\title{
CALIDAD DE VIDA RELACIONADA CON LA SALUD EN MUJERES OTOMÍES QUERETANAS
}

\author{
Rosalía Alonso Chombo, ${ }^{1}$ Jorge Adán Romero Zepeda ${ }^{2}$ e Hilda Romero Zepeda ${ }^{3}$
}

Resumen. Este artículo tiene como objetivo explorar las percepciones de los indígenas acerca de su salud y la forma en que reciben la atención médica. Esta investigación se vuelve relevante, ya que se identifica que hay muy pocos estudios en México que exploran la calidad de vida de los indígenas desde su propio marco interpretativo. Se parte del supuesto de que existe una discrepancia entre la visión biomédica que se tiene sobre la salud de los indígenas en el país, a la propia percepción que tienen estos grupos étnicos sobre su estado de bienestar físico, emocional, social y ambiental.

Palabras clave: indígenas, bioética, calidad de vida, salud, enfermedad.

\section{QUALITY OF LIFE RELATED TO HEALTH IN OTOMÍ WOMEN FROM QUERÉTARO}

Abstract. This article aims to explore the perceptions of indigenous about their health, and how they receive medical care. This research becomes relevant as it is identified that there are very few studies in Mexico that explore the indigenous Quality of Life from their own interpretive framework. It is assumed that there is a discrepancy between the biomedical point of view, from their self-perception that this ethnic groups have about their physical, emotional, social and environmental well-being.

Keywords: indigenous, bioethics, quality of life, health, illness.

\footnotetext{
${ }^{1}$ Doctorante. Docente- Investigadora Nivel VII de la Facultad de Contaduría y Administración, Campus Amealco Universidad Autónoma de Querétaro. Coordinadora del Campus Amealco. C.P.76803. rosalia.alonso@uaq.mx

${ }^{2}$ Profesor- Investigador de la Facultad de Contaduría y Administración, Universidad Autónoma de Querétaro, Campus Amealco. Sistema Nacional de Investigadores. C.P.76020 jorgeadan_r@yahoo.com

${ }^{3}$ Investigadora Nivel VII, Facultad de Ingeniería / Facultad de Derecho, Universidad Autónoma de Querétaro. Coordinadora de maestría en Bioética. C.P. 76060. Phd.hromero@gmail.com
} 


\section{1.- Introducción}

La característica más evidente que ha acompañado históricamente a los indígenas en México es su pobreza. Estos grupos comúnmente carecen de los recursos económicos suficientes como para satisfacer tan siquiera mínimamente sus necesidades básicas de alimentación, vivienda, trabajo formal, seguridad, educación escolarizada, o salud (Pineda, 2002: 260). Su poca incidencia en los espacios públicos se ve reflejada en falta de servicios domiciliares, y los entornos en los que habitan los hacen más vulnerables a contaminantes dañinos para la salud (Ávila y Velarde, 2002: 350).

Geográficamente, CONAPO identifica que, de 803 municipios con presencia predominantemente indígena, 407 son de alta y 300 de muy alta marginación, es decir, el 88\% viven por debajo de la línea de la pobreza (Torres et al, 2008: 809). En términos generales, la característica de pertenecer a un grupo indígena aumenta las probabilidades de ser pobre 4.5 veces más a la de la población mestiza. El paso de los años no mejora la situación; pues mientras que la tendencia de la población mestiza es mejorar de manera gradual sus condiciones de vida, entre los indígenas la pobreza se encuentra estancada (Méndez, 2010: 2).

Como es de suponerse, esta condición de los indígenas de pobreza y pobreza extrema los hace vulnerables a sufrir todo tipo de rezagos y desigualdades en materia de salud (Pelcastre, 2007: 1). La misma Organización Mundial de la Salud enfatiza que los indígenas están en desventaja con respecto del resto de la sociedad, siendo su estado de salud peor al del resto de los grupos de la sociedad (Méndez, 2010: 8) A grandes rasgos, en los indígenas encontramos más enfermedad y más muerte (Campos, 2009: 44).

Mientras que la mayoría población mestiza en México ha hecho la transición epidemiológica (mortalidad crónico-degenerativas principalmente), los indígenas suelen morir de enfermedades prevenibles (infecciosas, mala alimentación y violentas) (Torres et al, 2008: 810).

Entre las enfermedades infecciosas destacan las de transmisión sexual (por hábitos sexuales de riesgo); las intestinales (por condiciones insalubres y falta de agua potable); y las respiratorias (por falta de vivienda y vestimenta adecuadas) (Méndez, 2010: 2). La desnutrición está presente en la mayoría de los indígenas, principalmente por no disponer de los recursos económicos como para contar con la canasta básica (Campos, 2009: 44). 
Lejos de superar las enfermedades infecciosas, tal parece que entre los indígenas aumentan las probabilidades de morir por nuevas patologías surgidas de hábitos poco saludables: la diabetes por consumo de alimento chatarra y bebidas embotelladas; la cirrosis hepática por una mayor ingesta de bebidas embriagantes; y las enfermedades de transmisión sexual por encuentros íntimos con varias parejas sin hacer uso de preservativos (Amoroz, 2011: 22).

La esperanza de los indígenas con respecto a la población mestiza en México en promedio es 5 años menor. Los recién nacidos indígenas tienen una probabilidad de morir 3 veces mayor a la de los mestizos; del mismo modo, la muerte durante los 5 primeros años se duplica entre los indígenas con respecto a los mestizos (Méndez, 2010: 2).

Dentro de los grupos indígenas ya de por sí marginados, las mujeres viven en condiciones sociales que hacen que su calidad de vida en relación a la salud sea muy inferior a lo deseable, sobre todo en relación a las sobrecargas de trabajo doméstico. No suelen tener empleo remunerado, por lo que no tienen apoyo institucional para el cuidado de niños, enfermos y ancianos (cosa que lo tienen que hacer ellas); además, deben de atender animales de granja y cultivos de traspatio, todo esto por lo general sin contar con electrodomésticos ${ }^{4}$ que les aligeren la carga laboral (Sandoval, 2005: 162).

Oficialmente, estos problemas de salud agravados en la población indígena se justifican mediante la aseveración de que hay poca cobertura institucional en las zonas rurales porque estas se encuentran geográficamente muy extendidas, en comunidades pequeñas y de difícil acceso; sin embargo, lo que existe de fondo es un sistemático trato discriminatorio hacia estos grupos étnicamente diferenciados (Torres et al, 2008: 812).

Desde las instituciones gubernamentales se les excluye del desarrollo, dándose oportunidades desigualadas de acuerdo a la pertenencia cultural. El acceso a la salud a los indígenas está muy lejos de cubrir las recomendaciones hechas por los organismos internacionales, y esta inequidad distributiva está basada no en carencias presupuestales gubernamentales, sino en un simple y llano racismo (Méndez, 2010: 8).

En las clínicas de salud ubicadas en zonas indígenas, es común que el número de camas por habitante sea menor; el equipo médico sea obsoleto o inexistente; el inventario de

\footnotetext{
${ }^{4}$ Estufa, refrigerador, horno de microondas, lavadora de ropa, secadora de ropa, etc.
} 
medicamentos siempre se encuentre en situación de no haberse surtido en meses; o no exista servicio de ambulancia para trasladar a los enfermos graves a los hospitales.

Por otro lado, el personal de salud incurre sistemáticamente en las siguientes negligencias: deficiencias en el diagnóstico; atención de baja calidad y con alto grado de insatisfacción por parte de los derechohabientes; y seguimiento insuficiente a los tratamientos prescritos (Amoroz, 2011: 22).

Diversas investigaciones previas enfatizan que el personal de salud ubicado en clínicas rurales tratan con racismo a los indígenas, dándoles malos tratos de múltiples maneras: se les levanta la voz y se les habla con lenguaje altisonante cuando no hablan el español; se les amenaza con quitarles el beneficio de la asistencia médica; se les practican intervenciones invasivas sin consentimiento informado; y se les somete a prácticas biomédicas que no son sensibles a sus nociones culturales de pudor (Amoroz, 2011: 21; Torres et al, 2008: 812).

Como se podrá observar en los párrafos anteriores, la salud ha sido medida en indicadores tradicionales como morbilidad, mortandad y esperanza de vida (Tuesca, 2005: 80). Bajo estos instrumentos de medición, es fácil caer en la falacia economicista de que a mayor nivel socioeconómico, mayor salud; cuando en los hechos abundan los casos en donde grupos viviendo en condiciones de vida objetivamente peores que otros, sienten que tienen un mayor bienestar corporal (Moyano y Priego, 2009: 35).

Lo que se trata es precisamente romper con el paradigma dominante biomédico y mercantilista de las instituciones sanitarias oficiales, que consideran que la atención a la salud es un producto de consumo, en donde sólo importan los factores cuantitativos que se traducen el entradas y salidas de dinero (Fernández et al, 2010: 170).

Por lo tanto, se requiere otro marco interpretativo que contemple la salud y la enfermedad de manera más holística, tomando en cuenta elementos como el crecimiento personal, la realización de las potencialidades y el bienestar subjetivo; y como tal se propone el concepto de Calidad de Vida $^{5}$ (Ardila, 2003: 162).

\footnotetext{
${ }^{5}$ Como definición se entenderá calidad de vida como "Una medida compuesta de bienestar físico, mental y social, tal como la percibe cada individuo y cada grupo, y de felicidad, satisfacción y recompensa" (Ardila, 2003: 163).
} 
A grandes rasgos, Calidad de Vida es el conjunto de condiciones que contribuyen a hacer la vida agradable y valiosa (Fernández et al, 2010: 170). Antes que situaciones materiales, se entiende por el concepto de Calidad de Vida que los humanos tienen derechos para garantizar mínimamente el desarrollo potencialidades como su libertad, su libre albedrío, buenas relaciones sociales, seguridad e integridad física (del Amo, y Moctezuma, 2008: 2).

Se entiende por Calidad de Vida la capacidad de darse cuenta de su propia existencia, evaluándola a través de tanto sus propios estándares vivenciales, como de sus referentes culturales. Para establecerlo de manera cuantitativa, por lo general se toman en cuenta indicadores socioeconómicos tradicionales, más apreciaciones subjetivas sobre bienestar. (Urzúa y Caqueo, 2012: 64).

Como principal exponente del cual se desprende el Concepto de Calidad de Vida, se tiene al economista Amartya Sen, que afirma que para determinar el nivel de vida de un individuo no bastan los bienes materiales ni la utilidad que estos reporten, sino más bien establecer las condiciones sociales para que las personas puedan desarrollar sus capacidades.

Una mala calidad de vida se asociará con la pobreza, pero ser pobre no sólo es carecer de recursos económicos, sino sobre todo de sentir vergüenza, es decir, la sensación de que no se forma parte del bienestar generalizado, y que por ello las personas no pueden tener autoestima. Por ello, Calidad de Vida es poder cumplir las expectativas sociales mediante el derecho a acceder a bienes materiales a través de políticas públicas que lo hagan posible (Vera, 2005: 177).

Desde una perspectiva bioética, la calidad de vida es la que hace posible que un humano se desenvuelva con dignidad. Un agente adquiere la cualidad de autonomía moral en cuanto tenga la autopercepción de que su corporeidad y su entorno le permitan desarrollar libremente sus potencialidades y su felicidad.

Aludiendo a los principios básicos de bioética, se obtiene calidad de vida cuando hay autonomía, reflejada en su grado de satisfacción general hacia la vida, cuando se percibe que se están respetando los valores propios, y existe la posibilidad de su ejercicio de las generaciones futuras.

La justicia se vuelve también elemento de la calidad de vida, en tanto permite que se dé una distribución de los recursos sociales de una manera equitativa, así como se dé un 
respeto por el equilibrio ecológico. Finalmente, la beneficencia y la no maleficencia se entienden como imprescindibles para alcanzar la calidad de vida, ya que la convivencia pacífica se logra no solo mediante procurar no hacer daño intencional a otro, sino más bien ayudarlo a alcanzar su propia autorrealización (Asnariz, 2014: 4).

En aspectos de salud, cobrará importancia no sólo el hecho de contar o no con una enfermedad, sino de que existan canales institucionales y sociales que permitan externar la vivencia del estado físico, para que la irrupción a la cotidianidad de malestar sea haga pública, validada, y comience a ser curada desde el mismo momento en que sea "absuelta" de motivo de vergüenza (Urzúa et al, 2011: 139).

El desequilibrio físico es acompañado de una crisis emocional de ansiedad, miedo y desorientación, trastornando sus actividades físicas, laborales e interpersonales. Es a través del apoyo social e institucional es como se puede lograr la adaptación y superación a dicho acontecimiento vital (Vinaccia et al, 2008: 127).

Siendo así, la Calidad de Vida Relacionada con la Salud (CVRS) es un concepto relacionado al mismo tiempo con la salud y la enfermedad, con el bienestar social, la distribución equitativa de los recursos económicos del país, políticas públicas, valores culturales, la satisfacción de las necesidades humanas y los derechos positivos.

Así, para obtener información cuantificable sobre CVRS, se hace uso de indicadores multidimensionales que indaguen sobre los elementos sociodemográficos, las actividades asistenciales, la valoración de la atención por parte de los derechohabientes, y la percepción del mismo sujeto sobre las funciones de su cuerpo (Tuesca, 2005: 80).

Indicadores frecuentes son: el estado completo de bienestar físico, bienestar mental, disponibilidad de servicios de salud, y prevención de enfermedad mediante prácticas y entornos saludables (del Amo, y Moctezuma, 2008: 4).

La población total del municipio de Amealco de Bonfil, Querétaro en 2010 era de 62, 197 habitantes. De esta, 51, 295 habitantes (82\%) se encuentran ubicadas en localidades rurales (INEGI, 2010: 1; INEGI, 2011: 9). Amelaco de Bonfil es uno de los municipios del estado de Querétaro con mayor presencia indígena (27\%), comprendida principalmente por el grupo étnico otomí o ñahñu (99 \%) (INEGI, 2010: 2; INEGI, 2011: 10). 
Históricamente en México, la condición de indigenismo viene aparejada a la pobreza, así que en 2012 al población pobre del municipio representaba el 77\% y en pobreza extrema ${ }^{6}$ fue del 25\% (CENEVAL, 2012: 16).

Con respecto a los servicios de salud, datos oficiales reportan que el $75 \%$ de las personas en el municipio citado tiene derecho de a servicios médicos por parte de alguna institución pública o privada; siendo el servicio de Seguro Popular el más recurrido, atendiendo el 65.2\% de la población (INEGI, 2010: 1; INEGI, 2011: 10).

En la cabecera municipal, se pueden encontrar varias clínicas particulares, una clínica del Seguro Popular, otra del ISSSTE y otra a cargo del IMSS. Concretamente en San Idelfonso Tultepec, hay un centro de salud por parte del Seguro Popular, en donde se proporcionan consultas solamente de manera quincenal; en los hechos, en la mayoría de los casos los habitantes de la localidad de San Idelfonso Tultepec tienen que trasladarse a la cabecera municipal para atender su salud (Rivera, 2013: 56).

\section{2.- Materiales y Métodos}

Se realizó una encuesta a 10 mujeres de la comunidad del barrio de Xajay, Comunidad de San Idelfonso Tultepec municipio de Amealco de Bonfil, estado de Querétaro, México. La encuesta se basa en un instrumento validado y estandarizado por la Organización Mundial de la Salud (OMS) sobre Calidad de Vida Relacionada con la Salud (CVRS).

Este trabajo cuantitativo forma parte del diagnóstico comunitario participativo del proyecto de investigación-intervención para mejorar la alimentación del barrio de Xajay, emprendido por la Licenciatura en Desarrollo Local, Universidad Autónoma de Querétaro, plantel Amealco.

\section{3.- Resultados}

En la gráfica número 3.1 se muestra la edad de las mujeres entrevistadas por intervalos. 6 de 10 tienen edades comprendidas entre los 20 y los 39 años; 2 de 10 años su edad es de entre 40 y 49 años; y 2 de 10 tiene 50 años o más:

\footnotetext{
6 Por pobreza extrema se entenderá “Aquella que tiene un ingreso inferior a la línea de bienestar mínimo y tiene al menos tres carencias sociales”. (CENEVAL, 2012: 14)
} 


\section{1.- Edad}

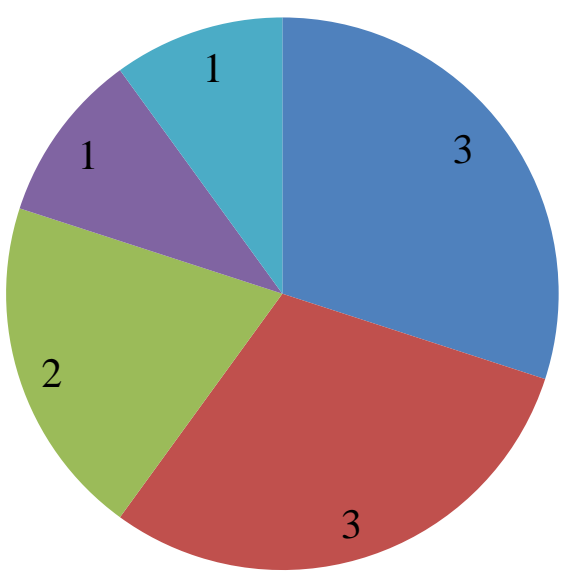

Entre 20 y 29 años

- Entre 30 y 39 años

Entre 40 y 49 años

- Entre 50 y 59 años

60 años o más

Fuente: Elaboración propia.

En la gráfica número 3.2 se muestra la presencia de enfermedades entre las personas del grupo. 8 de 10 se reconocen a sí mismas como sanas, mientras que dos de ellas tienen la enfermedad crónico-degenerativa de la diabetes:

\section{2.- Presencia de alguna enfermedad}

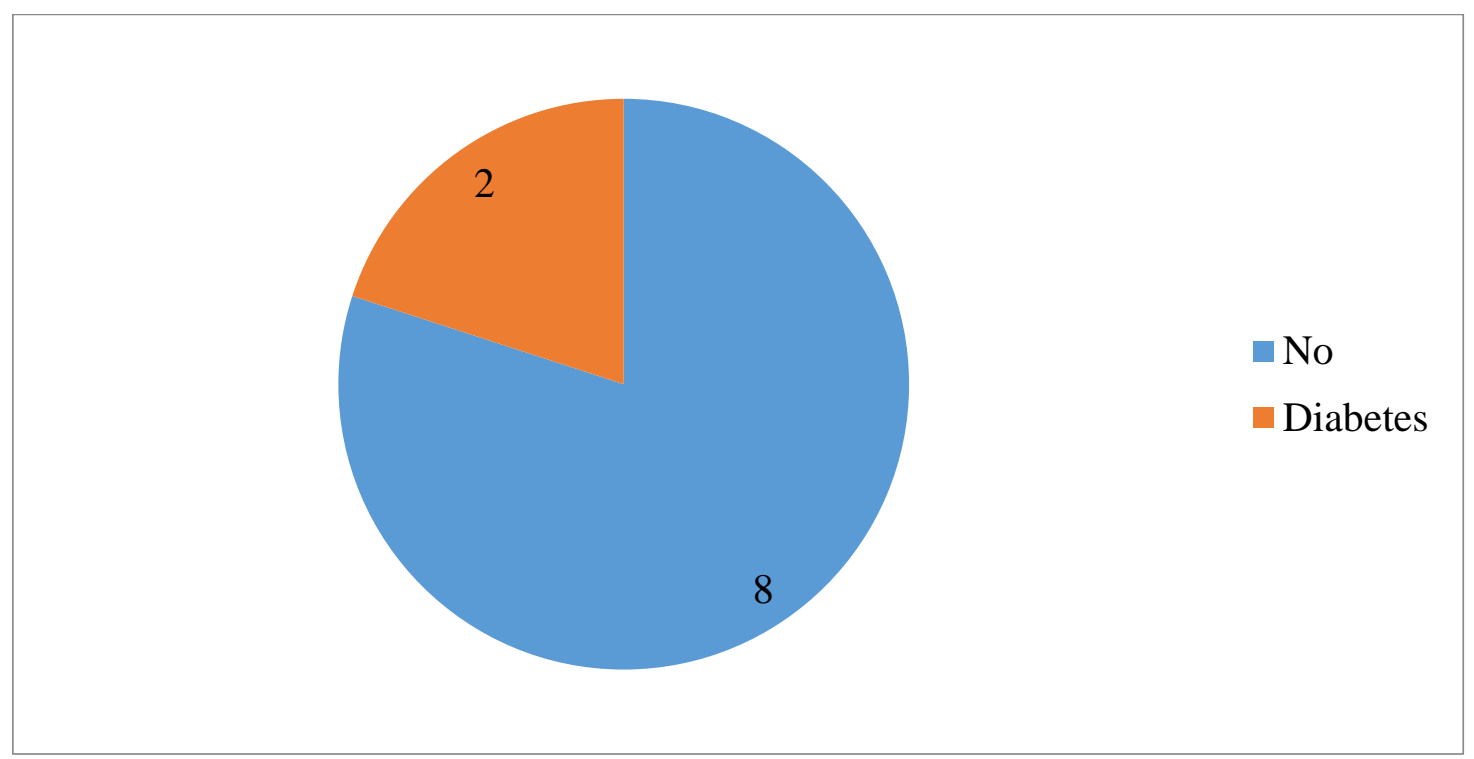

Fuente: Elaboración propia.

En la gráfica número 3.3 se muestra la cobertura de atención médica por parte de instituciones gubernamentales. 4 de 10 mujeres del grupo afirman no estar afiliadas a ninguna institución de salud: 


\section{3.- Atención médica}

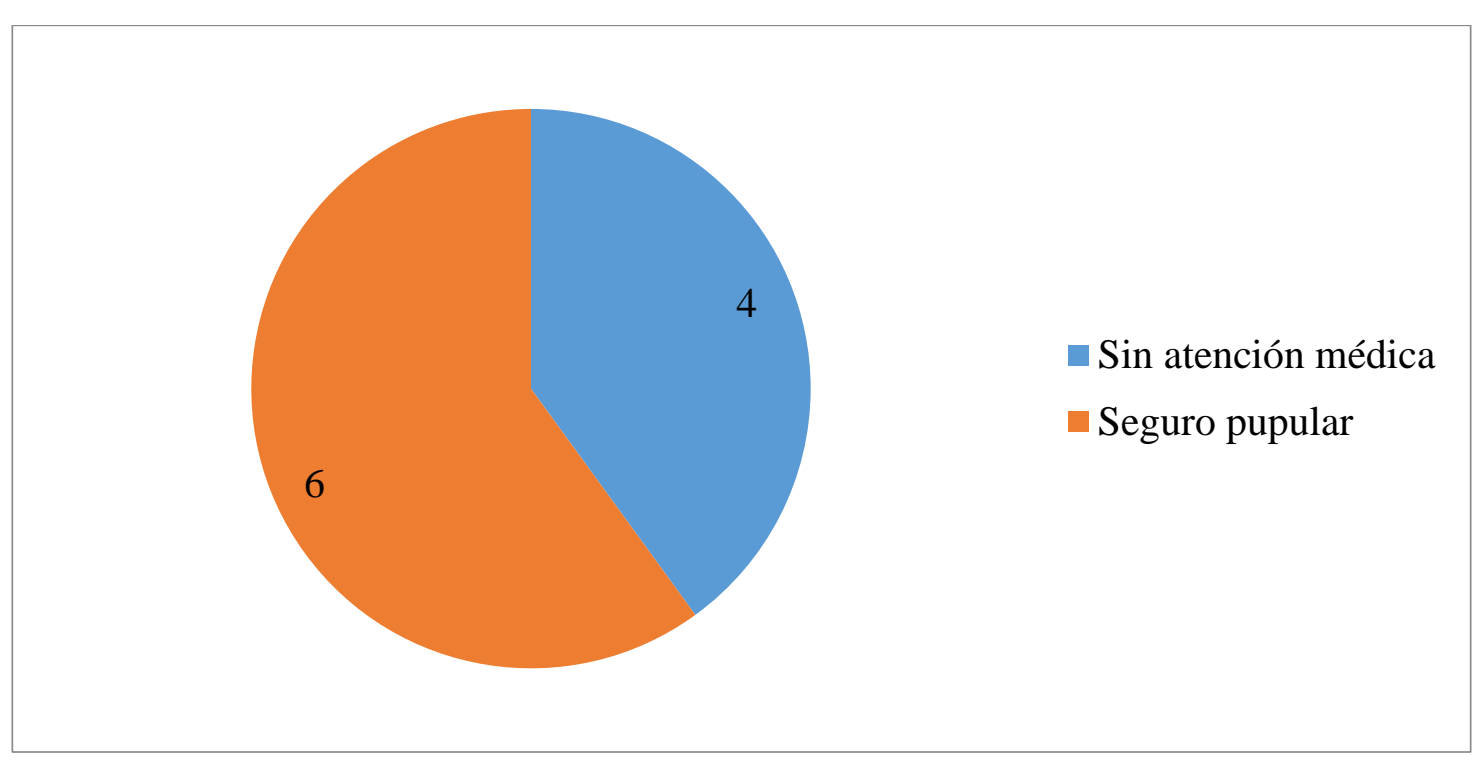

Fuente: Elaboración propia.

En la gráfica número 3.4 se muestra la percepción que se tiene sobre su propio estado físico. Las mismas dos mujeres con diabetes aseveran que su salud es lamentable, mientras que el resto oscila en sentir entre buena y muy buena salud:

\section{4.- Satisfacción sobre su estado de su salud}

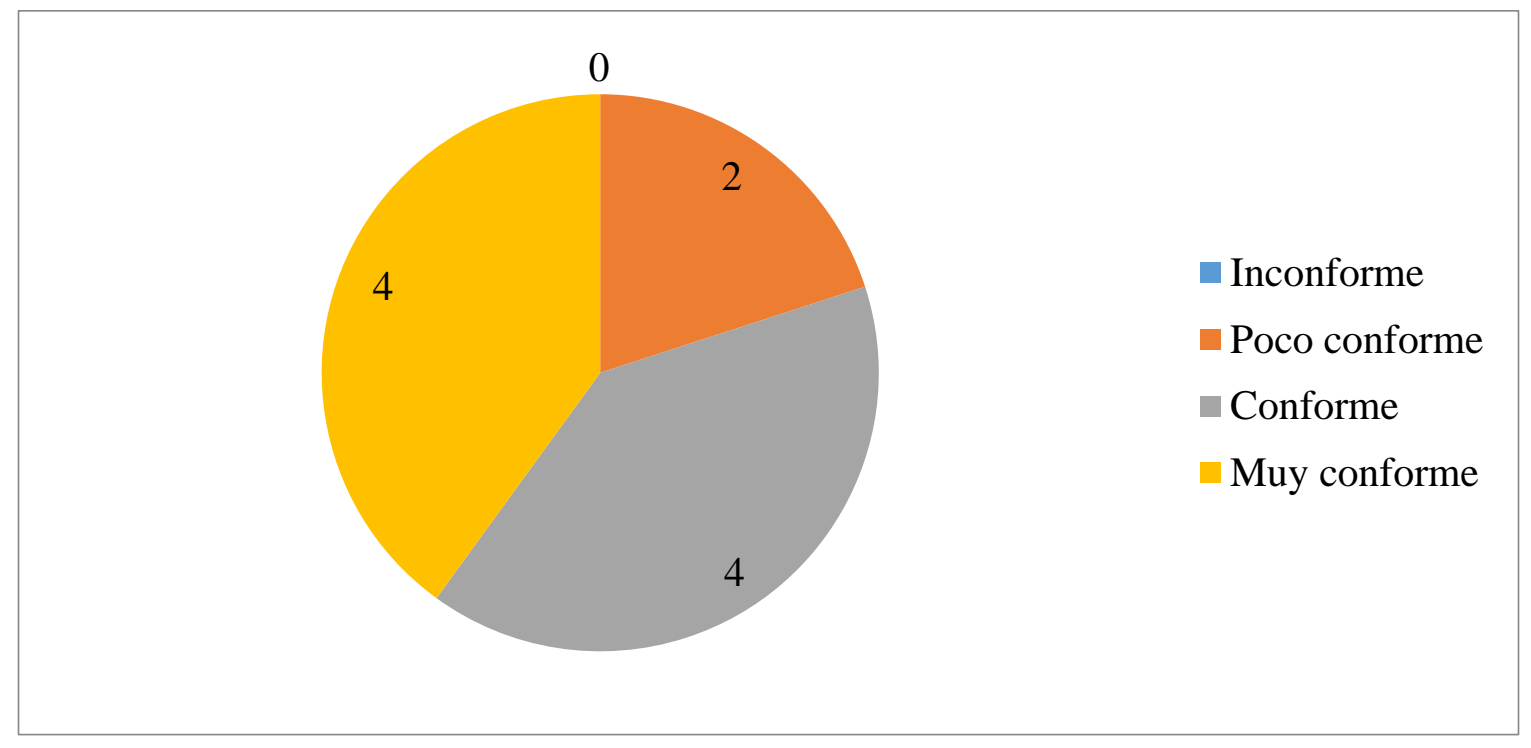

Fuente: Elaboración propia.

En la gráfica número 3.5 se muestra cuál es su percepción sobre el dolor físico. Destaca el hecho que, ante la presencia del malestar físico, 1 de 10 afirma que no puede hacer nada para aliviarlo, mientas que 6 de 10 sólo a veces mitiga el dolor: 


\section{5.- Percepción sobre el dolor físico}

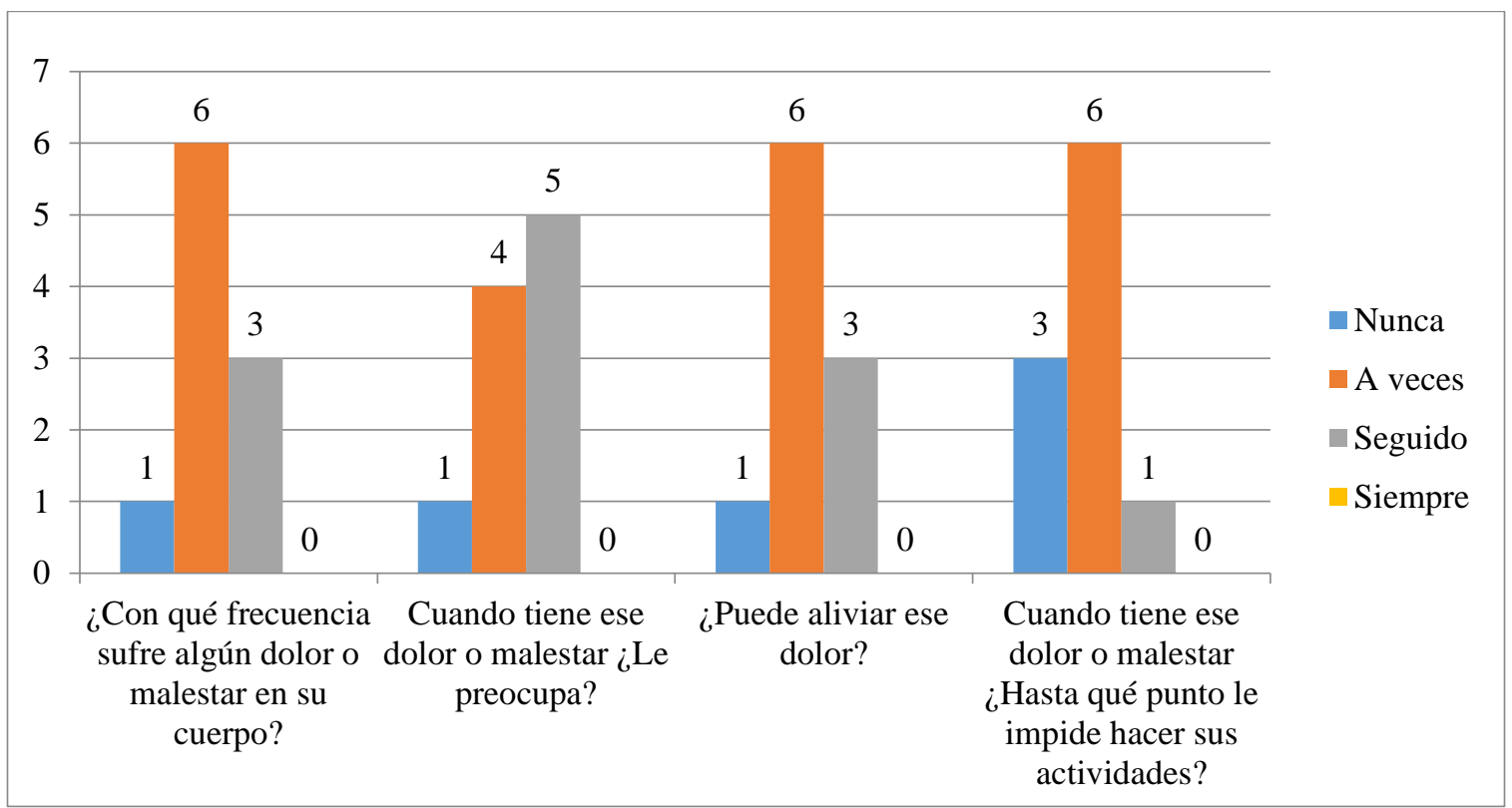

Fuente: Elaboración propia.

En la gráfica número 3.6 se muestra su apreciación sobre sus energías. Sobresale el que 9 de 10 termina a veces o siempre cansada al final del día, y que 6 de 10 piensa que nunca o a veces tiene fuerzas suficientes para el quehacer diario:

\section{6.- Percepción sobre vitalidad}

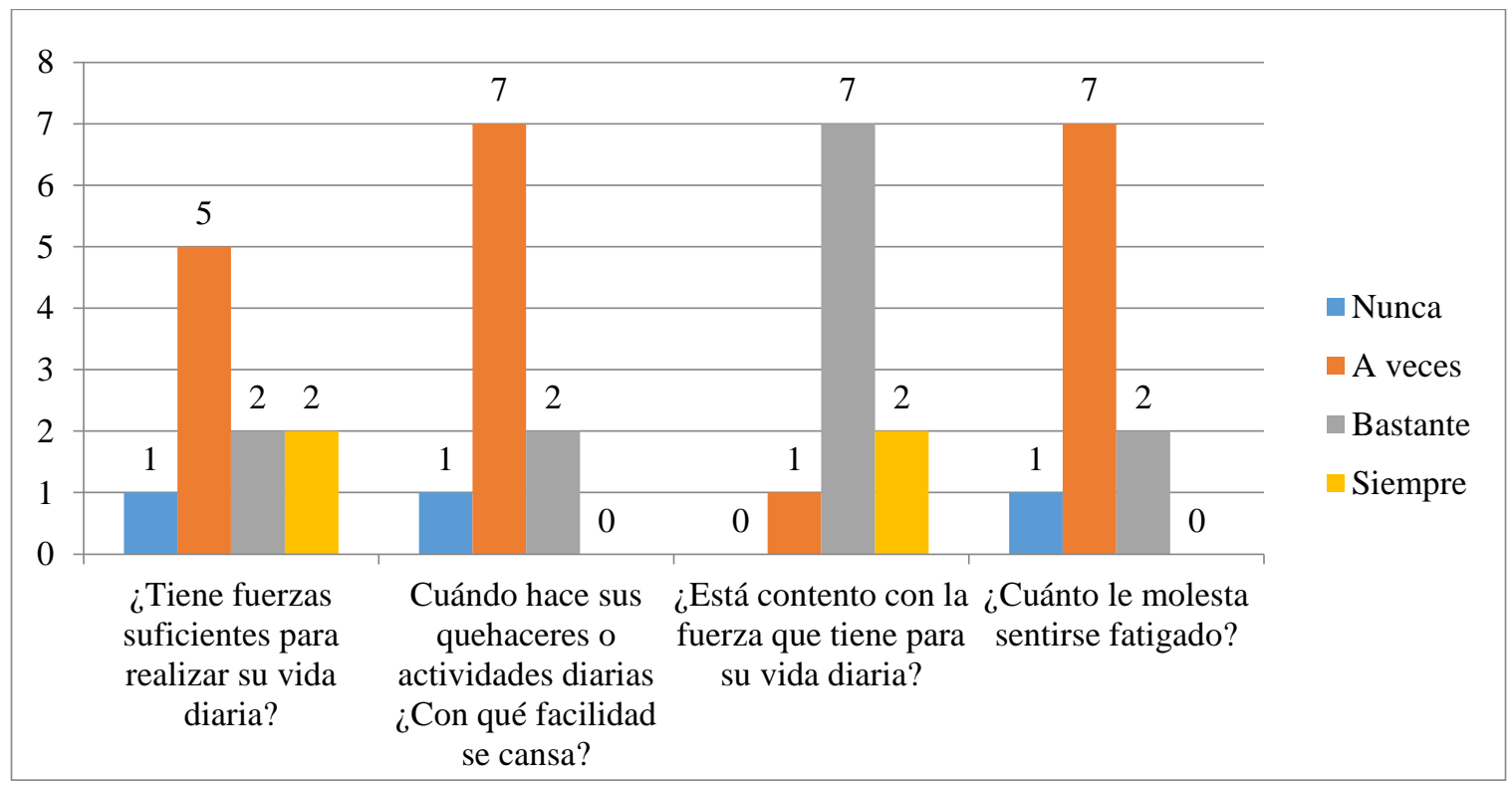

Fuente: Elaboración propia. 
En la gráfica número 3.7 se muestra la dependencia hacia los medicamentos. En general, un grupo de 7 de 10 afirma no necesitar medicina, mientras que, en diversos grados, 3 de 10 hace uso frecuente de drogas:

\section{7.- Uso de medicamentos}

\begin{tabular}{|c|c|c|c|c|c|}
\hline 9 & & & 8 & & \\
\hline 8 & & 7 & & & \\
\hline 7 & 6 & & & 6 & \\
\hline 5 & & & & & \\
\hline 4 & & & & 3 & \\
\hline 3 & 2 & 2 & 2 & & — Nada \\
\hline 2 & $\begin{array}{ll}1 & 1\end{array}$ & 1 & & 1 & — Poco \\
\hline 1 & & 0 & $\begin{array}{ll}0 & 0\end{array}$ & 0 & Regular \\
\hline & $\begin{array}{c}\text { ¿Para poder realizar } \\
\text { sus actividades } \\
\text { diarias ¿Qué tan } \\
\text { necesario es que } \\
\text { tome sus } \\
\text { medicamentos o } \\
\text { remedios? }\end{array}$ & $\begin{array}{c}\text { ¿Qué tanto necesita } \\
\text { de los medicamentos } \\
\text { o remedios para } \\
\text { realizar sus } \\
\text { actividades? }\end{array}$ & $\begin{array}{c}\text { Además de sus } \\
\text { medicamentos } \\
\text { ¿Cuánto depende de } \\
\text { otras cosas como } \\
\text { inyecciones, } \\
\text { curaciones, terapias, } \\
\text { para hacer su vida } \\
\text { diaria? }\end{array}$ & $\begin{array}{c}\text { Hasta qué punto su } \\
\text { calidad de vida } \\
\text { depende de los } \\
\text { tratamientos? }\end{array}$ & Mucho \\
\hline
\end{tabular}

Fuente: Elaboración propia.

En la gráfica número 3.8 se muestra su valoración sobre su disponibilidad de asistencia médica. En promedio, 6 de 10 considera que su salud es atendida de manera deficiente, en tanto a accesibilidad como en calidad:

\section{8.- Asistencia médica}

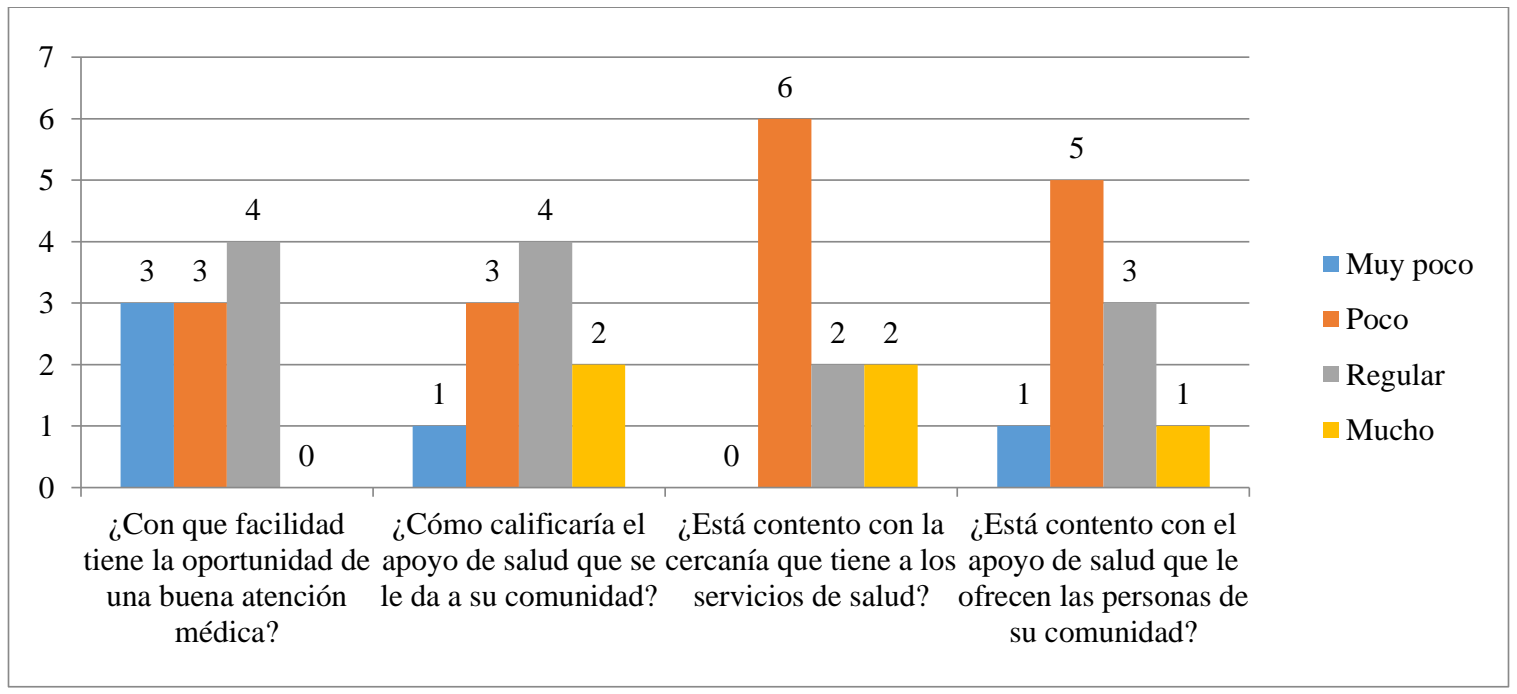

Fuente: Elaboración propia. 
En la gráfica número 3.9 se muestra su sensación emocional general. 3 de 10 señoras afirma que no disfruta la vida o sólo lo hace a veces, a su vez que 3 de 10 no se siente optimista o sólo lo en ocasiones:

\section{9.- Optimismo}

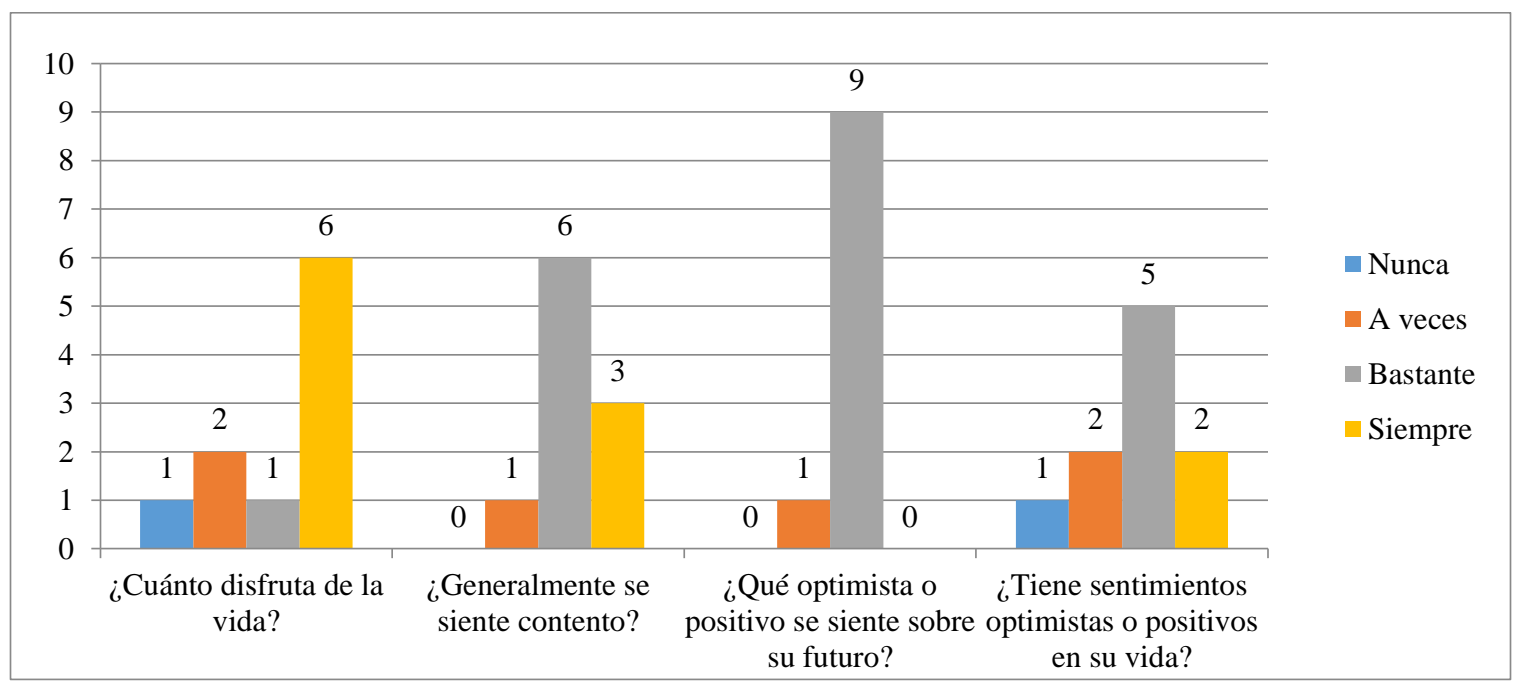

Fuente: Elaboración propia.

En la gráfica número 3.10 se muestra su autoevaluación sobre sus capacidades intelectuales. En términos generales, las mujeres en cuestión se sienten bastante competentes, ya que confían en su capacidad de aprender y tomar decisiones:

\subsection{0.- Percepción sobre capacidades intelectuales}

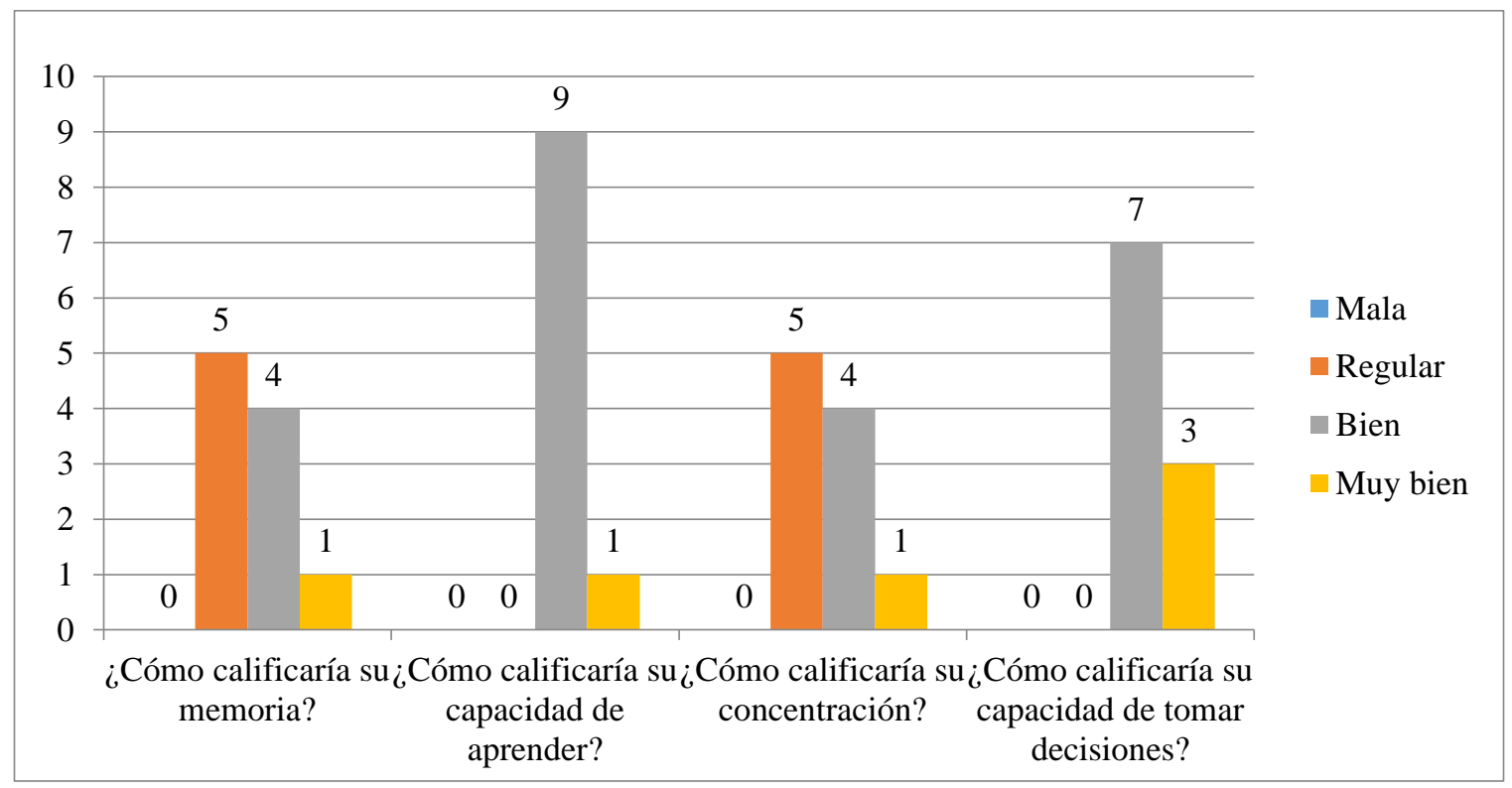

Fuente: Elaboración propia. 
En la gráfica número 3.11 se muestra su autoestima. En este aspecto, las entrevistadas muestran valores altos, siendo que se prácticamente todas se sienten seguras, capaces, contentas de sí mismas y altamente valoradas:

\subsection{1.- Autoestima}

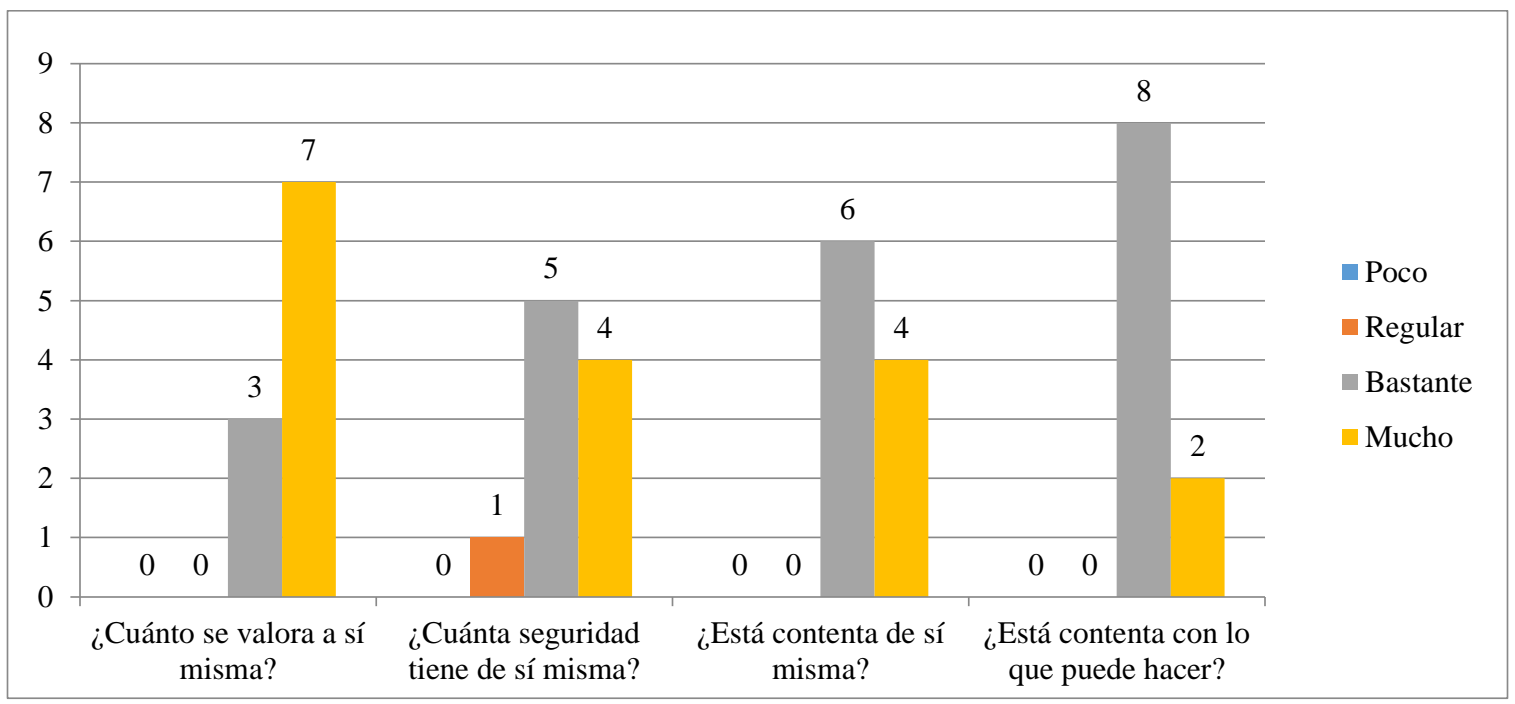

Fuente: Elaboración propia.

En la gráfica número 3.12 se muestra cuál es su aceptación sobre su propia apariencia.

De la misma manera, en todos los rubros casi toda la muestra se siente satisfecha con su imagen y con su cuerpo:

\section{12.- Aceptación de apariencia física}

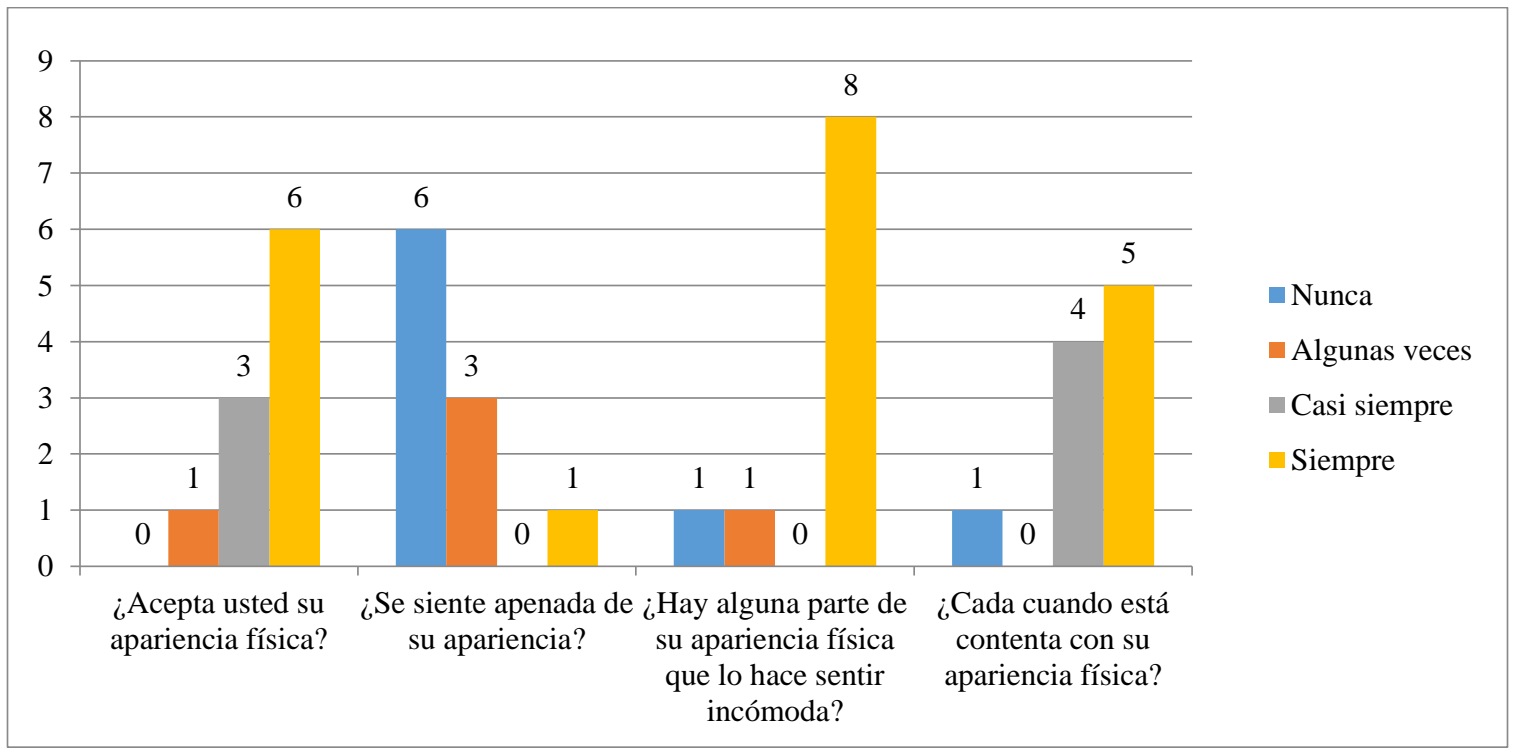

Fuente: Elaboración propia. 
En la gráfica número 3.13 se muestra su reconocimiento sobre su propensión a la depresión emocional. En términos generales, este malestar emocional está presente en 4 de 10 mujeres de la muestra en sus múltiples manifestaciones.

\subsection{3.- Depresión emocional}

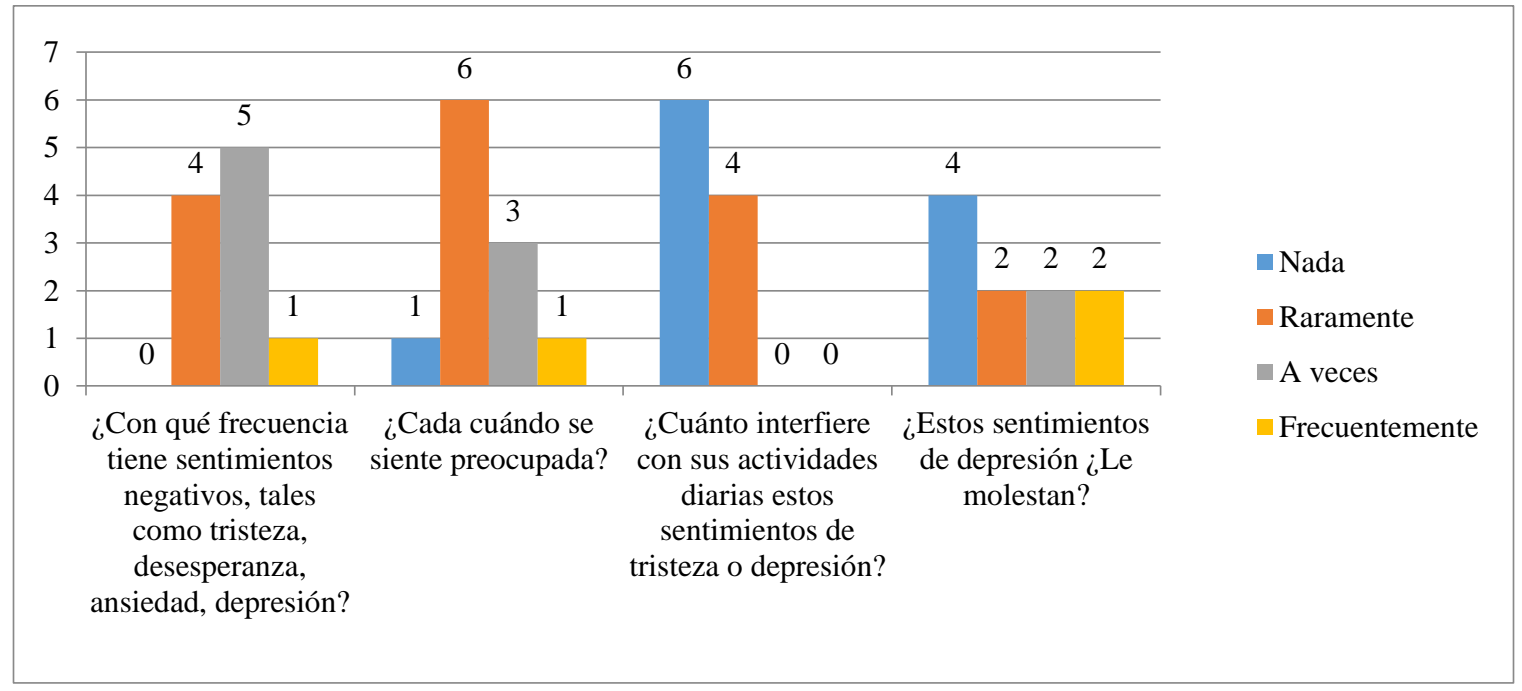

Fuente: Elaboración propia.

En la gráfica número 14 se muestra el cómo pueden reparar energías por medio del sueño. A pesar de que en casi todos los rubros no hay dificultades, 5 de 10 afirma que solo a veces el tiempo de dormir es suficiente para restablecerse:

\subsection{4.- Percepción sobre el sueño}

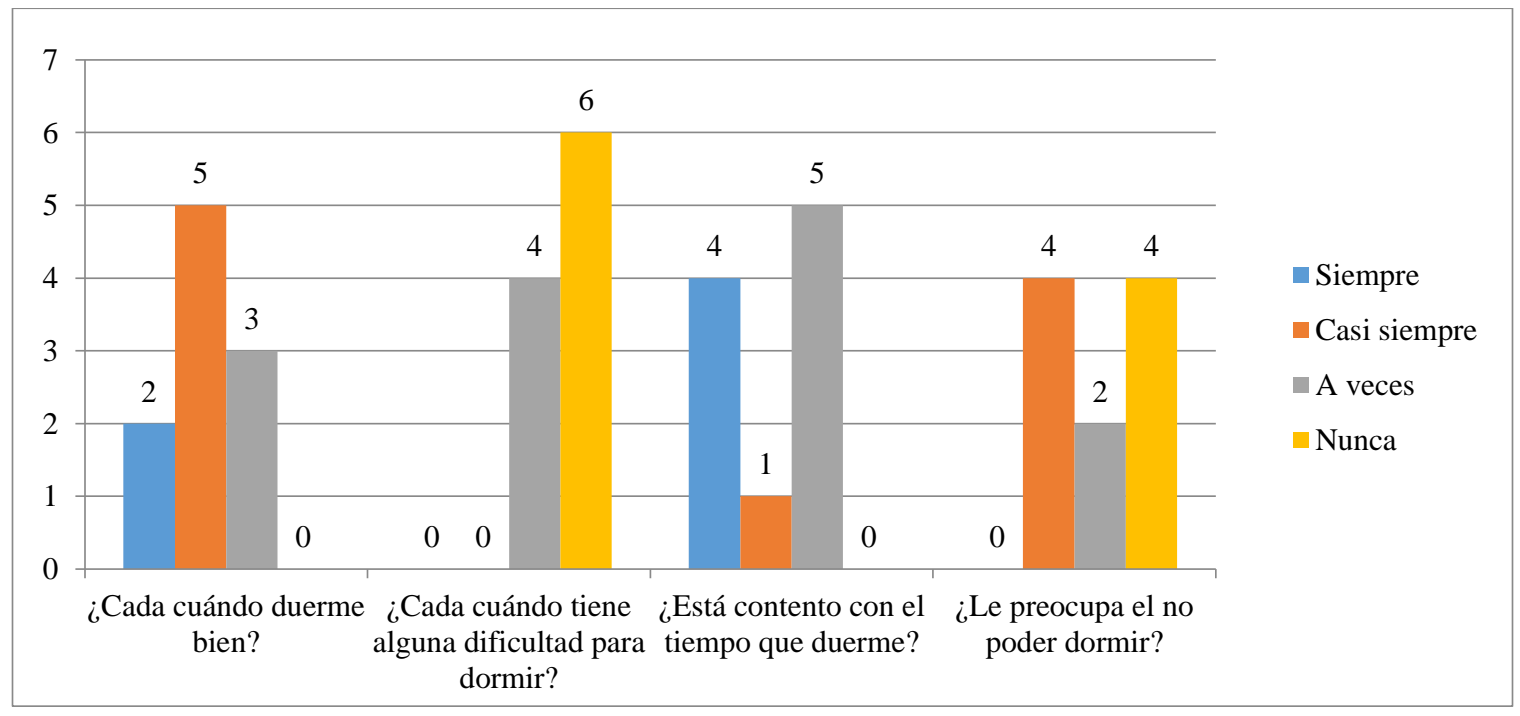

Fuente: Elaboración propia. 
En la gráfica número 15 se muestra su satisfacción sobre la vida íntima. En lo de satisfacción sobre las relaciones sexuales, un aproximado de 4 de 10 mujeres encuentra que estas son apenas satisfactorias o insatisfactorias:

\subsection{5.- Sexualidad}

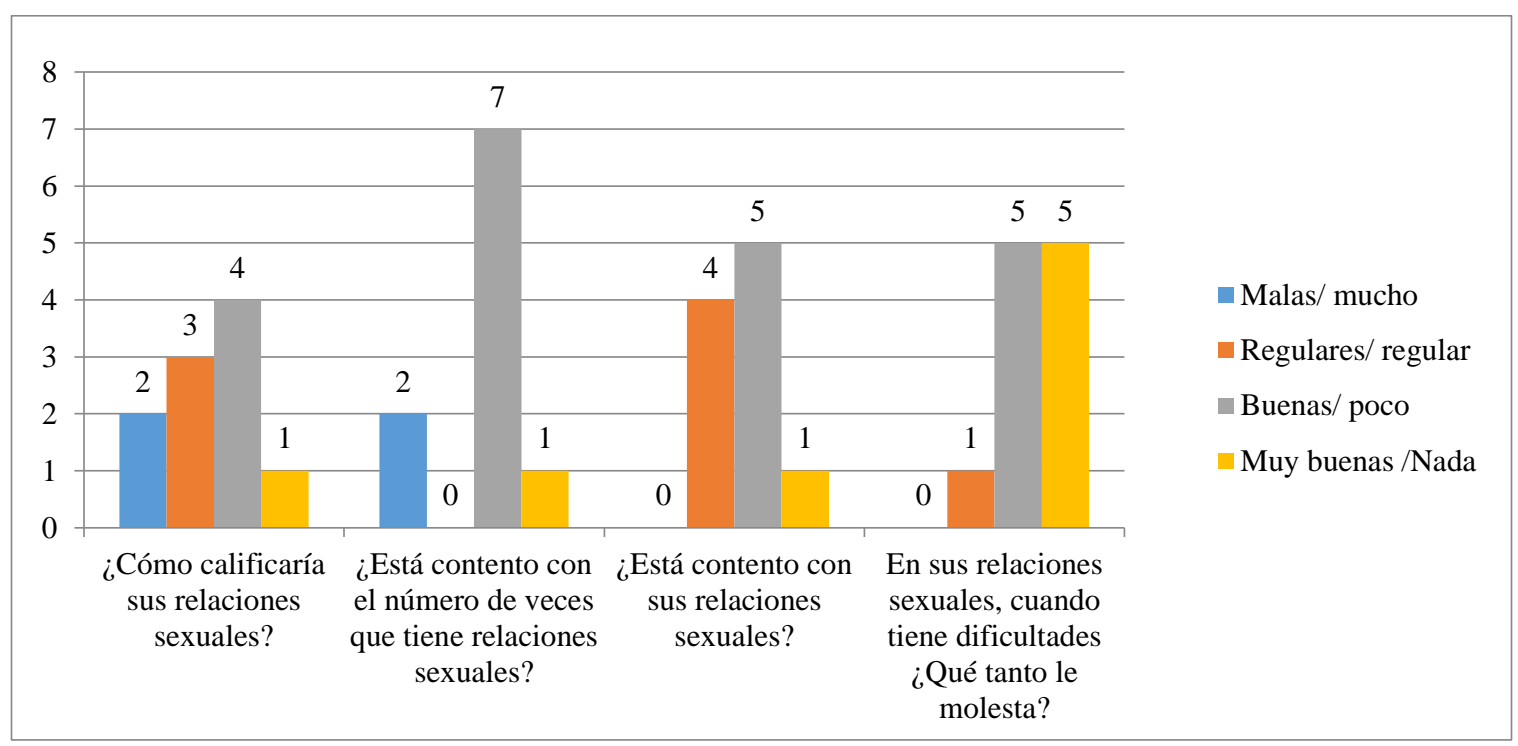

Fuente: Elaboración propia.

\section{4.- Conclusiones}

Por medio de la información obtenida en la muestra de entrevistadas, se puede inferir que en la población indígena otomí del estado de Querétaro existen tendencias significativas con respecto a su salud física, cobertura médica y salud emocional.

Tomando en consideración las gráficas de edad, presencia de enfermedad, satisfacción sobre su estado de salud y uso de medicamentos, observamos que las personas de más de 50 años aseveran que su salud es mala, y esto se debe principalmente a la presencia de enfermedades crónico degenerativas propias de las personas de edad avanzada.

En cambio, el grupo de entrevistadas de entre 20 y 49 años, juzga que su salud física no es motivo de preocupación.

Recuperando la información vertida en los gráficos atención médica, percepción sobre el dolor físico y asistencia médica, se encuentra que un aproximado de la mitad de las personas entrevistadas considera que su cobertura de salud es deficiente, no teniendo a quién recurrir ante la presencia de la enfermedad; incluso aproximadamente la mitad de la muestra 
percibe que, ante una situación de malestar físico, es muy azarosa la posibilidad de proporcionarse una atención adecuada.

Analizando la información de los cuadros percepción sobre vitalidad y percepción sobre el sueño, se concluye que las mujeres terminan sumamente cansadas al término del día, y que incluso el tiempo disponible para dormir no es lo suficiente como para convertirse en reparador.

Esto puede ser la manifestación de que las actividades que una mujer indígena promedio tiene que realizar día con día son físicamente muy demandantes, abrumadoras, y por ello, extenuantes; actividades productivas y reproductivas en demasía, que necesariamente, harán mella en su salud.

Retomando lo obtenido en las gráficas optimismo, percepción sobre sus capacidades intelectuales, autoestima, aceptación de apariencia física y depresión, se identifica que en el aspecto emocional las personas de la muestra tienen calificaciones altas, mostrando una gran aceptación de su vida, de su personalidad y de su apariencia física. No se distingue que en el entorno exista una propensión hacia las patologías psicológicas.

Por último, sobre el cuadro de sexualidad, destaca el hecho que esta no sea satisfactoria para casi la mitad de las entrevistadas. Para dar explicación a lo anterior, habría de agregarse de forma complementaria que el estado civil de 2 de 10 es viudez y 2 de 10 es abandonada.

Ante la falta de pareja y ante el rechazo social dentro de las comunidades otomíes de que las mujeres puedan darse satisfacción sexual con otras parejas que no sean sus esposos, es como esta ausencia de encuentros íntimos, ocasiona que este tema en particular les resulte insatisfactorio.

Los entornos en donde se desarrollan los indígenas otomíes son más saludables de lo que se suele suponer. Esta condición favorable al buen desarrollo físico y emocional se da sobre todo a las condiciones sociales comunales, que son calificadas como de convivencia estable, pacífica, donde todos se conocen entre sí, hay confianza entre ellos y se apoyan los unos a los otros (del Amo, y Moctezuma, 2008: 9).

Por el contario a lo que se suele asumir que en comunidades con un alto grado de industrialización o nivel socioeconómico alto necesariamente implica calidad de vida satisfactoria, desde un marco interpretativo de la bioética se cuestionaría que es precisamente 
en las sociedades estereotipadas por los medios masivos de comunicación occidentales como ideales, donde se suele presentar que hay una percepción de que la calidad de vida se encuentra deteriorada: baja autoestima, frustración de las capacidades, recelo para con la sociedad y sensación generalizada de infelicidad (Pareja, 2015: 2).

El problema de las comunidades indígenas surge en cuanto no hay apoyo institucional que vele de manera comprometida y sin discriminación la consecución de una Calidad de Vida en Relación a la Salud. Para ello, lo conveniente es que sean los mismos agentes los que diseñen su propia asistencia sanitaria; por su puesto auxiliándose del sector académico y biomédico, pero partiendo principalmente de sus propios pareceres y saberes de lo que es salud y enfermedad, tanto física, emocional, social, ambiental y espiritualmente (Campos, 2009: 45). 


\section{Referencias}

Amoroz, I. (2011) El derecho a la salud en comunidades indígenas del Estado de Chiapas. Revista Pueblos y Fronteras Digital, 6 (11) 8-37.

Ardila, R. (2003) Calidad de vida: una definición integradora. Revista Latinoamericana de Psicología, 35(2) 161-164.

Asnariz, T. (2014, mayo) ¿Cómo relacionamos el aspecto inmunológico con la calidad de vida y bioética? Jornadas de Psicoinmunología. Trabajo presentado en Jornadas de Psicoinmunología, Mar del Plata, Argentina.

Ávila, C. y Velarde, E. (2002) Evaluación de la calidad de vida. Salud Pública de México, 44 (4) 349-

361.

Campos, R. (2009) Los retos de la Salud Intercultural en México. Aquí estamos. Revista de exbecarios indígenas del IFP-México. Miradas indígenas sobre la salud pública. 6

(11) 44-51.

CENEVAL (2012) Informe de pobreza y evaluación en el estado de Querétaro 2012, Distrito Federal, Consejo Nacional de Evaluación de la Política de Desarrollo Social.

Del Amo, S. y Moctezuma, S. (2008) Bienestar o calidad de vida, en una comunidad de la sierra totonaca de Veracruz. Iberóforum. Revista de Ciencias Sociales de la Universidad Iberoamericana, 3 (5) 1-18.

Fernández, J., Fernández, M., Cieza, A. (2010) "Los conceptos de calidad de vida, salud y bienestar analizados desde la perspectiva de la clasificación internacional de funcionamiento (CIF)" Revista Española de Salud Pública 2010; 84 (2) 169-

184.

INEGI (2010) Censo de población u vivienda 2010. Estado de Querétaro. Información por municipio. Amealco de Bonfil. Aguascalientes. Instituto Nacional de Estadística y Geografía.

INEGI (2011) Panorama sociodemográfico de Querétaro. Aguascalientes. Instituto Nacional de Estadística y Geografía.

Méndez, R. (2010) Condiciones de vida y salud en zonas indígenas de Yucatán, México: 1990 y 2005. Población y Salud en Mesoamérica, 8 (1)1-18.

Moyano, E. y Priego, C. (2009) Marco teórico para analizar las relaciones entre paisaje natural, $\quad$ salud y calidad de vida. Sociedad Hoy, (16) 31-44.

Pareja, J. (2015) ¿Qué entiende la bioética por calidad de vida? Usos y abusos del concepto. Fundación Bioética. Consultado el 13 Julio de 2015. Disponible en: http://www.bioeticacs.org/?dst=cvida

Pelcastre, B, (2007) La salud de los pueblos indígenas: acciones y políticas. Salud Pública de México, 49, 83.

Pineda, S. (2002) La mujer indígena: ante la pobreza. Convergencia. Revista de Ciencias Sociales, 9 (28) 251- 264.

Rivera, A. (2013) Jóvenes, ritos de paso y educación en el rincón de San Idelfonso Tultepec, Amealco, Querétaro.Querétaro. Universidad Autónoma de Querétaro.

Sandoval, A. (2005) Pobreza y género en los indígenas contemporáneos. Revista Argentina de Sociología, 3 (5) 156-171.

Torres, K., Arredondo, A., Duarte, M. y Madrid, V. (2008) "La mujer indígena, 
vulnerable a cáncer cérvicouterino: Perspectiva desde modelos conceptuales de salud pública", en Salud en Tabasco, 14 (3) 807-815.

Tuesca, R. (2005) La calidad de vida, su importancia y cómo medirla”, en Salud Uninorte, (21) $76-86$.

Urzúa, A. y Caqueo, A. (2012) Calidad de vida: Una revisión teórica del concepto. Terapia Psicológica, 30 (1) 61-71.

Urzúa, A., Pavlov, R., Cortés, R., Pino, V. (2011) "Factores Psicosociales Relacionados con la Calidad de Vida en Salud en Pacientes Hemodializados" Terapia Psicológica, vol. 29, núm. 1, julio, 2011, pp. 135-140,

Vinaccia, S., Fernández, H., Quiceno, J., López, M., Otalvaro, C. (2008) “Calidad de Vida Relacionada con la Salud y Apoyo Social Funcional en Pacientes Diagnosticados con VIH/Sida", en Terapia Psicológica, vol. 26, núm. 1, julio, 2008, pp. 125-132.

Vera, R. (2005) "Elementos constitutivos para medir la pobreza y la calidad de vida", en Espacios Públicos, vol. 8, núm. 15, febrero, 2005, pp. 176-189. 